\title{
CONSTRUCTING PRIME-FIELD PLANAR CONFIGURATIONS
}

\author{
GARY GORDON
}

\begin{abstract}
An infinite class of planar configurations is constructed with distinct prime-field characteristic sets (i.e., configurations represented over a finite set of prime fields but over fields of no other characteristic). It is shown that if $p$ is sufficiently large, then every subset of $k$ primes between $p$ and $f(p, k)$ forms such a set (where $f(p, k)=2^{\left[\left(\sqrt{p}-A k^{3 / 2}\right) / B k^{3 / 2}\right]}$ for constants $A$ and $B$ ). In particular, for every positive integer $k$, there exist infinitely many planar matroid configurations $C_{i, k}$ with $\left|\chi_{p f}\left(C_{i, k}\right)\right|=k$ (where $\chi_{p f}(C)$ denotes the prime-field characteristic set of $C)$. We also give a result concerning cofinite prime-field characteristic sets.
\end{abstract}

1. Introduction. We are interested in the problem of constructing finite configurations $C$ of points and lines such that $C$ can be represented precisely over fields of given characteristics, where the characteristics have been previously specified. More precisely, for a planar configuration $C$ of points and lines, define the characteristic set $\chi(C)$ to be a set of primes (perhaps including zero) such that $p \in \chi(C)$ if and only if there is some field $F$ of characteristic $p$ and some subset $C^{\prime}$ of the projective plane $P G(2, F)$ such that $C$ and $C^{\prime}$ have the same incidences. Let $P=\{0,2,3,5, \ldots\}$ denote the set of all field characteristics. Then $\chi \subseteq P$ is a characteristic set if $\chi=\chi(C)$ for some configuration $C$. Finally, if there is a $C$ such that $p \in \chi(C)$ implies $C$ can be embedded in $P G(2, p)$, we call $\chi$ a prime-field characteristic set, and denote it $\chi_{p f}(C)$ or $\chi_{p f}$.

Since such finite configurations are planar matroids, we can restate the above definitions in terms of matroid representation theory. In these terms, $p \in \chi(C)$ if there is a field $F$ of characteristic $p$ and a rank three matrix $M$ with entries in $F$ such that:

(1) There is a one-to-one correspondence between the points of $C$ and the columns of $M$.

(2) Three points are collinear in $C$ if and only if the corresponding three columns of $M$ are linearly dependent.

In what follows, we use the terms "matroid", "configuration" and "matroid configuration" interchangeably to refer to a rank three matroid. In addition, we use "point" (of $C$ ) and "column" (of $M$ ) interchangeably, when no confusion can arise.

Characteristic sets for matroids were formally defined by Ingleton [6], while prime-field sets were introduced by Brylawski [1]. The study of possible characteristic sets, however, appears implicitly in the work of Pappus, Pascal, Descargues and

Received by the editors September 23, 1982 and, in revised form, September 27, 1983.

1980 Mathematics Subject Classification. Primary 05B35, 51E20, 51D20.

Key words and phrases. Matroid configuration, characteristic set, prime-field characteristic set. 
Fano. Subsequently, it has been shown that:

(1) $0 \in \chi(C) \Rightarrow \chi(C)$ is cofinite (Rado [8]).

(2) $\chi(C)$ infinite $\Rightarrow 0 \in \chi(C)$ (Vamos [11]).

(3) Every cofinite characteristic set (necessarily including 0 ) is realizable (Reid unpublished but see [2]).

(4) All finite characteristic sets (necessarily excluding 0) are realizable (Kahn [7]).

The questions concerning both finite and cofinite characteristic sets were solved using fields which contain many transcendentals. Thus the corresponding questions about prime-field sets remain open.

It is well known that $\chi_{p f}(P G(2, p))=\{p\}$, and so all singletons in $P$ (except $\{0\})$ form prime-field sets. Reid [9] exhibited the first two element prime-field set when he constructed a configuration $C$ with $\chi_{p f}(C)=\{1103,2089\}$. Brylawski and Reid [2] generalized these techniques to construct many finite, nonsingleton primefield sets. (Reid's example was the first two element characteristic set, as well.)

The main result of this paper, Theorem 4.1, says that for every $k>0$, every subset of primes $p_{1}<p_{2}<\cdots<p_{k}$ with $p_{1}$ sufficiently large and

$$
p_{k}<2^{\left[\left(\sqrt{p_{1}}-A k^{3 / 2}\right) / B k^{3 / 2}\right]}
$$

for fixed constants $A$ and $B$, independent of $k$ and $p_{1}, \ldots, p_{k}$, forms a prime-field characteristic set. Thus, for all $k>0$, there are infinitely many prime-field sets containing exactly $k$ primes.

§2-4 below are concerned with constructing finite prime-field characteristic sets.

Finally, we thank Professor Tom Brylawski for his helpful conversations and Professor William Lenhart for computing assistance.

2. Background construction. Our goal in $\$ \S 2-4$ is to construct finite prime-field characteristic sets. To determine $\chi$ or $\chi_{p f}$ for a given configuration $C$, it is useful to construct a representing matrix (when $\chi \neq \varnothing$ ) in a canonical way.

Definition 2.1. Two matrices $A_{1}$ and $A_{2}$ over $F$ are projectively equivalent if one can be obtained from the other by a sequence of the following operations:

(1) elementary row operations,

(2) multiplication of columns by nonzero scalars,

(3) an automorphism of $F$,

(4) removal of a row of zeros.

Since each of these operations preserves column dependences, projectively equivalent matrices represent isomorphic matroid configurations. A matroid $C$ (with $\chi(C) \neq \varnothing$ ) is projectively unique if any two matrices which represent it (over $F$ ) are projectively equivalent. Finally, a matroid $C$ is sequentially unique if there is an ordering of the points of $C=\left\{x_{1}, \ldots, x_{n}\right\}$ such that for each $1 \leqslant i \leqslant n$, the submatroid $\left\{x_{1}, \ldots, x_{i}\right\}$ is projectively unique.

For planar configurations (rank 3 matroids), this means that we can assume the first four points of $C$ form a quadrangle (a matroid circuit), and each successive point is on at least two lines generated by previous points. For such configurations, determining $\chi$ amounts to examining all subdeterminants of the representing matrix. 
Given $k$ primes $p_{1}<p_{2}<\cdots<p_{k}$ with $k \geqslant 2$, we will construct a configuration which satisfies two conditions.

I. All but the primes $p_{1}, \ldots, p_{k}$ are eliminated from $\chi_{p f}$.

II. No prime $p_{1}, \ldots, p_{k}$ itself was eliminated in the process.

To satisfy condition I, we will construct a configuration $C$ such that any matrix representing $C$ (over the prime $p_{1}$ ) will contain a subdeterminant equal to a nonzero multiple of $p_{1} \cdots p_{k}$. This is accomplished by using the von Staudt calculus and results of [3] to do arithmetic synthetically. We then introduce a dependence which forces this product to be zero. This will eliminate all characteristics not dividing $p_{1} \cdots p_{k}$, and thus satisfy I. Condition II, however, requires more careful work.

Although much of what follows is quite algebraic, the reader should keep in mind the underlying geometric structure. We are only concerned with representing pointline incidences with the column dependences of a matrix.

Let $p_{1}<p_{2}<\cdots<p_{k}$ be our finite set of primes and set $N=p_{1} \cdot p_{2} \cdots p_{k}+2$. Let $b_{i}$ denote the integer represented by the first $i$ digits of the binary expansion of $N$. Thus, $b_{1}=1, b_{2}=2$ or 3 , and, in general, $b_{i+1}=2 b_{i}$ or $2 b_{i}+1$. This is the general binary construction in [1].

In [1], this $b_{i}$ sequence is used in constructing a sequentially unique planar configuration $C$ satisfying I above (i.e., $\chi_{p f}(C) \subseteq\left\{p_{1}, \ldots, p_{k}\right\}$ ). Among other features, this configuration has one line containing distinct points corresponding to each $b_{i}\left(1 \leqslant i \leqslant \log _{2} N+1\right)$. Thus, for example, if $p_{1}=5$ and $p_{2}=7$ (with $k=2$ ), then $N=37$ and this line contains six points corresponding to the six $b_{i}$ values $1,2,4,9,18,37$. But $b_{3}=4 \equiv 9=b_{4}(\bmod 5)$, and so these two points form a multiple point (a two-point circuit) over the prime 5 , a dependence not replicated over 7. Thus II above is not satisfied in this example.

We circumvent this problem in two ways. First, we modify the $b_{i}$ sequence to avoid certain "bad" values, e.g., $b_{i} \equiv 0\left(\bmod p_{1}\right)$ but $b_{i} \not \equiv 0\left(\bmod p_{2}\right)$. Second, we use projective cross ratios to preserve the $b_{i}$ coordinate values from one line to another, so the resulting configuration will not contain a "bad" line, as above. This avoids the problem $b_{i} \equiv b_{j}\left(\bmod p_{1}\right)$ but $b_{i} \not \equiv b_{j}\left(\bmod p_{2}\right)$.

In Figure 2.2 below, each line $l_{i}$ will contain points with coordinates involving a modification of our $b_{i}$ sequence. These lines all meet at the point 0 . The points $P_{i}$ are used to project certain points from $l_{i}$ to $l_{i+1}$ by preserving specified cross ratios.

We now modify the $b_{i}$ sequence. Let $\bar{b}_{i}=b_{i}$ for $i=1,2$ and 3 . In general, we define $\bar{b}_{i}$ inductively. Consider the following matrix $M(i)$ :

$$
\left[\begin{array}{cccccccccccc}
1 & 0 & 0 & 1 & 1 & 0 & 1 & 1 & 0 & 1 & 0 & 0 \\
0 & 1 & 0 & 1 & 1 & 1 & -r_{i-1} & 0 & 1 & 2 & 1 & 1 \\
0 & 0 & 1 & 1 & 0 & 1 & 0 & r_{i-1} & \bar{b}_{i-1} & \overline{b_{i}} & \overline{b_{i}} & \bar{b}_{i-2}
\end{array}\right],
$$

where $\bar{b}_{i}=2 \bar{b}_{i-1}+r_{i-1}$ and $i \geqslant 4$. (M(i) will be a fundamental building block in our construction. For now, we use its $3 \times 3$ subdeterminants to determine which possible values for $\bar{b}_{i}$ are to be avoided.) 
Now consider all $3 \times 3$ subdeterminants of $M(i)$ involving the term $\bar{b}_{i}$. There are at most $\left(\begin{array}{c}12 \\ 3\end{array}\right)=220$ such subdeterminants, and many of these will not involve $\bar{b}_{i}$. In any case, let $D$ be the constant denoting the number of subdeterminants involving $\bar{b}_{i}$ in a nontrivial way.

We assume inductively that $\bar{b}_{1}, \bar{b}_{2}, \ldots, \bar{b}_{j-1}$ have all been defined so that:

(*) None of the $D$ subdeterminants of $M(i)$ vanish $\left(\bmod p_{m}\right)$ for any $m$.

We also assume, for $i<j$,

A. $\left|\overline{b_{i}}-b_{i}\right| \leqslant D \cdot k$,

B. $\bar{b}_{i}=2 \bar{b}_{i-1}+r_{i-1}$, where $\left|r_{i-1}\right| \leqslant 3 D \cdot k+1$.

Now define $\bar{b}_{j}$ to be the integer closest to $b_{j}$ so that $(*)$ holds for $M(j)$. (If this choice is not unique, we arbitrarily select the larger value.)

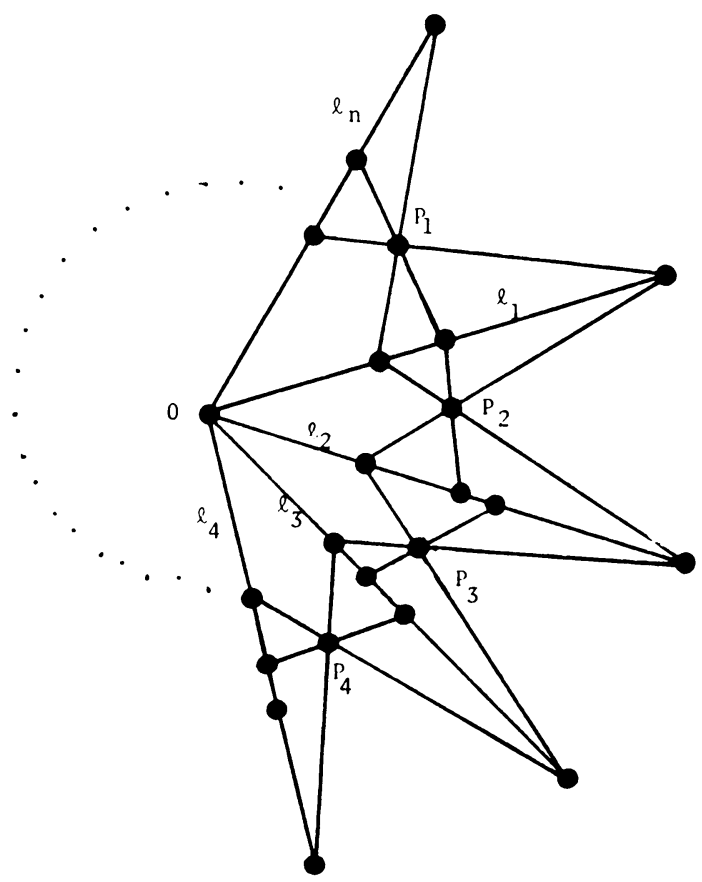

FIGURE 2.2

Lemma 2.3. Suppose $6 D \cdot k<p_{1}<\cdots<p_{k}$. Then the integer $\bar{b}_{j}$ defined above satisfies $\mathrm{A}$ and $\mathrm{B}$.

Proof. For condition A, we note that each of the $D$ subdeterminants of $M(j)$ involving $\bar{b}_{j}$ is at most a quadratic expression in $\bar{b}_{j}$ (since the first row of $M(j)$ contains no $\bar{b}_{j}$ or $r_{j-1}$ terms). Thus any subdeterminant $S$ involving $\bar{b}_{j}$ nontrivially can be congruent to zero $\left(\bmod p_{m}\right)$ for some $m$ for at most $2 k$ distinct integers near $b_{j}$ (i.e., in the interval $\left[b_{j}-D \cdot k, b_{j}+D \cdot k\right]$ ). Thus, at most $2 k \cdot D$ potential choices for $\bar{b}_{j}$ have been ruled out. Since $\bar{b}_{j}$ is the integer closes to $b_{j}$ avoiding these 
(at most) $2 k \cdot D$ choices, we must have $\left|\bar{b}_{j}-b_{j}\right|<D \cdot k$ and A is satisfied. For $\mathrm{B}$, we have

$$
\begin{aligned}
\left|\bar{b}_{j}-2 \bar{b}_{j-1}\right| & \leqslant\left|\bar{b}_{j}-b_{j}\right|+\left|b_{j}-2 b_{j-1}\right|+2\left|b_{j-1}-\bar{b}_{j-1}\right| \\
& \leqslant D \cdot k+1+2 D \cdot k=3 D \cdot k+1 .
\end{aligned}
$$

This completes the proof.

3. Construction of the matrix $M(N)$. As before, assume $6 D \cdot k<p_{1}<\cdots<p_{k}$ and set $N=p_{1} \cdots p_{k}+2$. Define the $\bar{b}_{i}$ sequence as in the previous section. We will not construct a matrix whose column dependences over $p_{1}$ give a configuration with characteristic set $\left\{p_{1}, \ldots, p_{k}\right\}$.

In Part 1 below, we construct a matrix whose coordinates contain all the possible values $r_{i}$ can assume (where $\bar{b}_{i+1}=2 \bar{b}_{i}+r_{i},\left|r_{i}\right| \leqslant 3 D \cdot k+1$ ). Appropriate $r_{i}$ values will then be preserved by projecting corresponding points onto lines created in Part 2.

\begin{tabular}{|c|c|c|c|c|c|c|c|c|c|c|c|c|c|c|c|c|}
\hline$a_{2}$ & $a_{3}$ & $a_{4}$ & $a_{5}$ & $a_{6}$ & $a_{7}$ & $a_{8}$ & $a_{9}$ & $a_{10}$ & $a_{11}$ & $a_{12}$ & & & & & & $a_{3 t+6}$ \\
\hline 0 & 0 & 1 & 1 & 1 & 0 & 1 & 2 & 1 & 1 & 1 & 1 & 1 & 1 & 1 & 1 & 1 \\
\hline 1 & 0 & 1 & 1 & 0 & 1 & -1 & 0 & 2 & 2 & -2 & 3 & 3 & -3 & $t$ & $t$ & $-t$ \\
\hline 0 & 1 & 1 & 0 & 1 & 1 & 0 & 1 & 1 & 0 & 0 & 1 & 0 & 0 & 1 & 0 & 0 \\
\hline
\end{tabular}

PART 1. $r_{i}$ submatrix. Let $t=3 D \cdot k+1$ and let $M_{1}$ be

If we take column dependences of $M_{1}$ over $p_{1}$, thus creating a matroid configuration $C_{1}$, we get the following proposition.

Proposition 3.1. $C_{1}$ is sequentially unique.

Proof. Results of [3] allow us to assume columns $a_{1}-a_{4}$ are uniquely determined. For $i \geqslant 4$ we list the two dependences which determine each column $a_{i}$ uniquely over the integers (and hence over the prime subfield of any field).

$$
\begin{array}{lll}
a_{5}: a_{1} a_{2}, a_{3} a_{4} & a_{10}: a_{2} a_{4}, a_{5} a_{7} & \\
a_{6}: a_{1} a_{3}, a_{2} a_{4} & a_{3 i-1}: a_{1} a_{2}, a_{3} a_{3 i-2} & \text { for } 4 \leqslant i \leqslant t+2, \\
a_{7}: a_{1} a_{4}, a_{2} a_{3} & & \\
a_{8}: a_{1} a_{2}, a_{6} a_{7} & a_{3 i}: a_{1} a_{2}, a_{9} a_{3 i-2} & \text { for } 4 \leqslant i \leqslant t+2, \\
a_{9}: a_{1} a_{3}, a_{4} a_{8} & a_{3 i+1}: a_{2} a_{4}, a_{7} a_{3 i-1} & \text { for } 4 \leqslant i \leqslant t+1 .
\end{array}
$$

PART 2. $\bar{b}_{i}$ submatrices. We wish to construct a matrix whose coordinates contain our $\bar{b}_{i}$ sequence. Consider the matrix $M_{2}(i)$ for $1 \leqslant i \leqslant n\left(\right.$ where $\left.\bar{b}_{n+1}=b_{n+1}=N\right)$ :

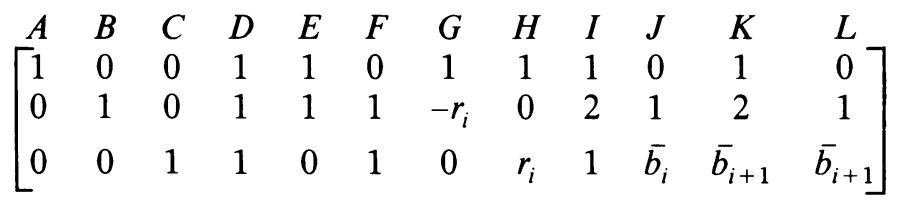

(Compare with matrix $M(i)$ in §2.) Note that $M_{2}(1)$ (with columns $G$ and $J$ deleted) gives a sequentially unique matroid (over $p_{1}$ or over the integers). In general, $M_{2}(i)$ will have all its coordinates uniquely determined once columns $G$ and $J$ have been determined. 


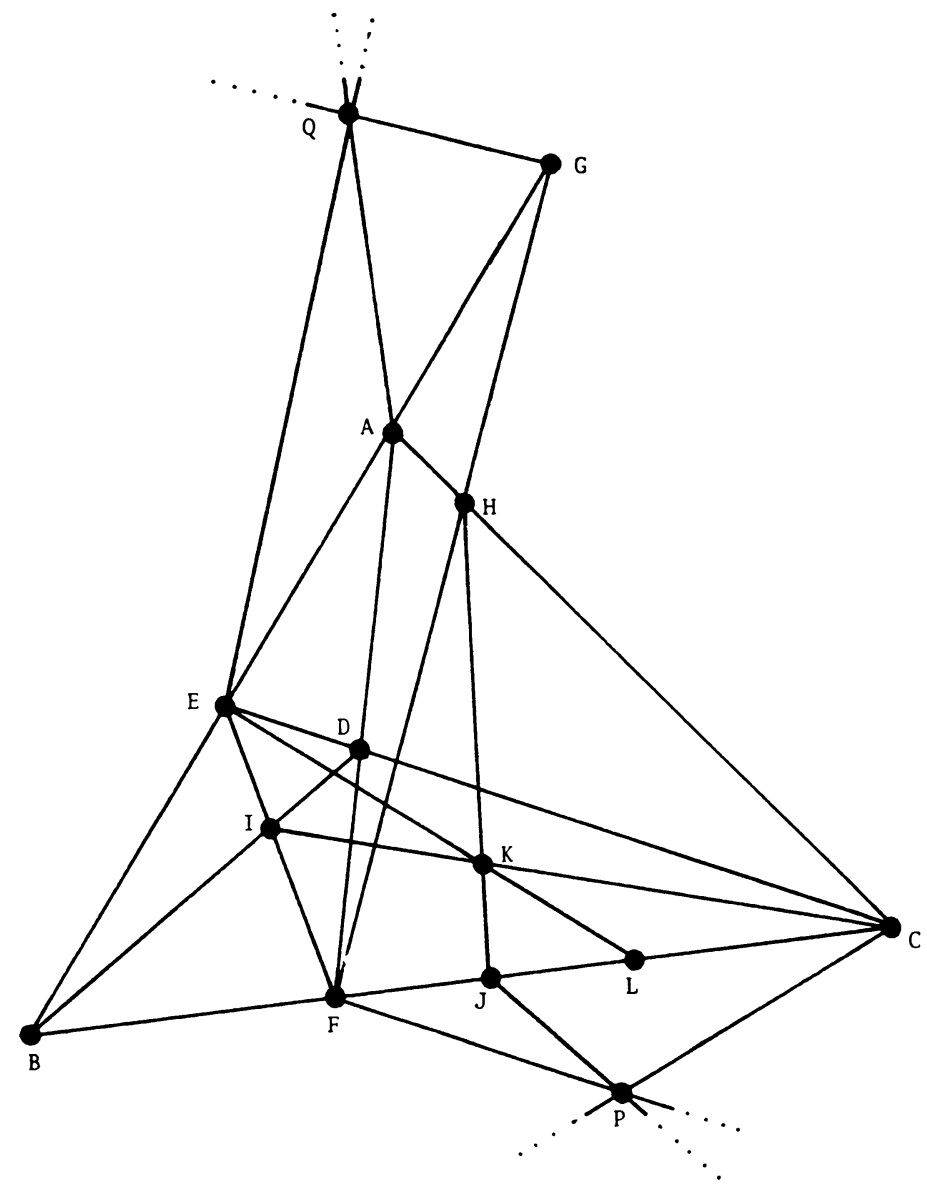

FIGURE 3.2

Our basic idea is to "recode" $M_{2}(i)$ by introducing a transcendental $x_{i}$ in the coordinates for $A, B$, and $D$. Column $G$ will be determined by projecting the appropriate $r_{i}$ value from the line $z=0$ in $M_{1}$, while column $J$ will be determined (for $i>1$ ) by projecting the point corresponding to column $L$ in $M_{2}(i-1)$. The configuration resulting from this is pictured in Figure 3.2.

More precisely, let $x_{1}, \ldots, x_{n}$ be independent transcendentals, where $\bar{b}_{n+1}=N$ is the last term in the $\bar{b}_{i}$ sequence. For $1 \leqslant j \leqslant n$, we define the "recoded" matrix $M\left(x_{j}\right)$ recursively. Assume $M\left(x_{i}\right)$ has been defined for $i<j$ and consider the columns $A, C$ and $D$ in the submatrix $M_{2}(j)$. Replace column

$$
A \text { by }\left[\begin{array}{c}
1 \\
x_{j} \\
x_{j}
\end{array}\right], \quad C \text { by }\left[\begin{array}{c}
1 \\
0 \\
x_{j}^{2}
\end{array}\right], \quad D \text { by }\left[\begin{array}{c}
1 \\
x_{j} \\
1
\end{array}\right]
$$


and call these replacements $A_{j}, C_{j}$ and $D_{j}$ respectively. (Column $B$ (which is column $a_{2}$ of the submatrix $M_{1}$ ) is unchanged.) Then $E_{j}-L_{j}$ are determined uniquely as in Figure 3.2. For example, column $F_{j}$ is on the line $\overline{a_{2} C_{j}}$ and $\overline{A_{j} D_{j}}$, so

$$
F_{j}=\left[\begin{array}{c}
1 \\
x_{j} \\
x_{j}^{2}
\end{array}\right]
$$

The others follow similarly. We note, as mentioned above, that $G_{j}$ and $J_{j}$ may not have been uniquely determined when $j>1$. To accomplish this, we add two points, $Q_{j}$ and $P_{j}$, to $M\left(x_{j}\right)$ when $j>1$ as follows: $Q_{j}$ is on $\overline{a_{1} A_{j}}$ and $\overline{a_{5} E_{j}}$ and $P_{j}$ is on $\overline{C_{j-1} C_{j}}$ and $\overline{F_{j-1} F_{j}}$ (see Figure 3.2). In addition, we add column $P_{1}$ to $M\left(x_{n}\right)$ where $P_{1}$ is determined by $\overline{a_{3} C_{n}} \cap \overline{a_{7} F_{n}}$. Further, since $H_{j}$ can be uniquely derived without the points $Q_{j}$ and $G_{j}$ precisely when $\left|r_{j}\right| \leqslant 1$, we delete columns $Q_{j}$ and $G_{j}$ from $M\left(x_{j}\right)$ when $\left|r_{j}\right| \leqslant 1$; otherwise, we leave them in. Finally, add $L_{1}^{\prime}$ to $M\left(x_{1}\right)$ if $\bar{b}_{2}=3$, where $L_{1}^{\prime}$ is on $\overline{a_{2} C_{1}}$ and $\overline{D_{1} K_{1}}$. $\left(L_{1}^{\prime}\right.$ corresponds to

$$
\left[\begin{array}{l}
0 \\
1 \\
2
\end{array}\right]
$$

in $M_{2}(1)$.)

We put Parts 1 and 2 together to define the matrix $M(N)=\left[M_{1} M\left(x_{1}\right) M\left(x_{2}\right)\right.$ $\left.\cdots M\left(x_{n}\right)\right]$. Then $M(N)$ is a $3 \times K$ matrix, where $K \approx 14 \cdot \log _{2} N+9 D \cdot k+9$. Thus, in $M(N)$, given our (unjustified) choices for $A_{j}, C_{j}$ and $D_{j}$, our remaining coordinates are uniquely determined. After our earlier concern about sequential uniqueness, the reader may be distressed by the obvious lack of same above. We remark, however, that we can still determine the characteristic set (of the derived configuration) by simply examining subdeterminants of $M(N)$. This follows from the fact that no numerical values have been assigned gratuitously in $M(N)$, i.e., if some subdeterminant is zero (or nonzero) over a field $F$, this reflects an actual dependence (or independence) encountered in trying to embed the derived configuration in $P G(2, F)$.

THEOREM 3.3. Suppose $6 D \cdot k<p_{1}<\cdots<p_{k}$ and let $C(N)$ be the configuration arising from the column dependences of $M(N)$ over the prime $p_{1}$. Then $\chi(C(N))=$ $\left\{p_{1}, \ldots, p_{k}\right\}$.

Proof. We first show that $\chi(C(N)) \subseteq\left\{p_{1}, \ldots, p_{k}\right\}$. Now the three columns $L_{1} L_{n} P_{1}$ (or $L_{1}^{\prime} L_{n} P_{1}$ if $\bar{b}_{2}=3$ ) have determinant equal to

$$
\left|\begin{array}{ccc}
2 & N & 1 \\
x_{1} & x_{n} & 0 \\
2 x_{1}^{2} & N x_{n}^{2} & -x_{1} x_{n}
\end{array}\right|=(N-2) x_{1} x_{n}\left(x_{1}+x_{n}\right)=p_{1} \cdots p_{K} \cdot x_{1} x_{n}\left(x_{1}+x_{n}\right) \text {. }
$$

Thus $L_{1}$ (or $L_{1}^{\prime}$ ), $L_{n}$ and $P_{1}$ are collinear in $C(N)$ (since $p_{1}$ divides this determinant). Since the factor of $p_{1} \cdots p_{k}$ is uniquely determined (see arguments preceding Theorem 3.3), these three points will be independent over characteristic $p$ for $p \neq p_{i}$, $1 \leqslant i \leqslant k$. Thus $\chi \subseteq\left\{p_{1}, \ldots, p_{k}\right\}$. 
It remains to show the reverse inclusion. This requires a systematic check of all subdeterminants. For each $2 \leqslant m \leqslant k$, we must show the column dependences of $M(N)$ are exactly the same over $p_{m}$ as they are over $p_{1}$. Now since each $p_{m}>6 D \cdot k$, any subdeterminant in which no $\overline{b_{i}}$ term appears will remain the same when considered modulo the respective primes (as $6 D \cdot k \geqslant$ the absolute value of some coefficient in any such subdeterminant). Therefore, it suffices to consider only those subdeterminants which involve at least one of $J_{i}, K_{i}$, or $L_{i}$.

Case 1. Subdeterminants in which all three columns are in one $M\left(x_{i}\right)$. These correspond to "coded" versions of the subdeterminants of $M(i)$ from $\$ 2$. For example, $\operatorname{det}\left(D_{i} G_{i} L_{i}\right)=\left(x_{i}^{3}-x_{i}\right) \cdot S$, where $S$ is the corresponding subdeterminant in $M(i)$. By construction of the $\bar{b}_{i}$ sequence and since $x_{i}$ is transcendental, these subdeterminants cause no trouble. If $P_{i}$ or $Q_{i}$ is in our subdeterminant, see Case 2.

Case 2. Not all three columns are in any $M\left(x_{i}\right)$. We sketch the proof of Case 2. A more detailed analysis of such subdeterminants is left to the reader.

First note that any $3 \times 3$ subdeterminant not containing any $P_{i}$ or $Q_{i}$ will simply be a polynomial in $x_{1}, \ldots, x_{n}$ with at least one nonzero coefficient. For example,

$$
\operatorname{det}\left(J_{i} A_{j} a_{3 r+5}\right)=\left|\begin{array}{ccc}
\overline{b_{i}} & 1 & 1 \\
x_{i} & x_{j} & r \\
\overline{b_{i}} x_{i}^{2} & x_{j} & 0
\end{array}\right|=r \overline{b_{i}} x_{i}^{2}+x_{i} x_{j}-\overline{b_{i}} x_{i}^{2} x_{j}-r \overline{b_{i}} x_{j}
$$

is never zero modulo any of $p_{1}, \ldots, p_{k}$.

Including $P_{i}$ may give rise to a subdeterminant of the form $f\left(\bar{b}_{r}, \bar{b}_{s}\right) \cdot g\left(x_{i}, x_{j}\right)$ for nonconstant polynomials $f$ and $g$. But this subdeterminant can only occur when $j=i-1$ and $|r-s| \leqslant 2$. This follows from the fact that points on the line $\overline{a_{2} C_{i-1}}$ are projected onto the line $\overline{a_{2} C_{i}}$ via $P_{i}$ (see Figure 2.2). Thus our $3 \times 3$ subdeterminant above must contain one point on $\overline{a_{2} C_{i-1}}$ and on $\overline{a_{2} C_{i}}$. Evaluating the resulting determinants yields expressions which are zero over each $p_{m}$ or nonzero over each $p_{m}$. For example, $\operatorname{det}\left(L_{i} P_{i} J_{i-1}\right)=x_{i} x_{i-1}\left(x_{i}+x_{i-1}\right)\left(\bar{b}_{i+1}-\bar{b}_{i-1}\right)$. But $\bar{b}_{i+1}-\bar{b}_{i-1}$ is a subdeterminant of $M(j)$ from $\S 2$, with $j=i+1$, and so the construction of the $\overline{b_{i}}$ sequence precludes problems here.

The story is similar for the point $Q_{i}$, although slightly easier. In either case, any subdeterminant $S \equiv 0\left(\bmod p_{m}\right)$ for some $m$ will have $S \equiv 0\left(\bmod p_{m}\right)$ for all $m$. This concludes our proof.

4. Finite prime-field characteristic sets. In Theorem 3.3, we note that although $\left\{x_{1}, \ldots, x_{n}\right\}$ were chosen to be transcendental, all that was needed was a guarantee that certain determinants involving these variables did not vanish. Indeed, the construction will remain valid as long as these variables do not satisfy any member of the finite list of polynomials arising from all the subdeterminants of $M(N)$. This observation leads to our main theorem.

THEOREM 4.1. Suppose

$$
6 D \cdot k<p_{1}<\cdots<p_{k}<2^{\left[\left(\sqrt{p_{1}^{-}}-A k^{3 / 2}\right) / B k^{3 / 2}\right]}=f\left(p_{1}, k\right),
$$


where $A, B$ and $D$ are fixed constants (independent of $k, p_{1}, \ldots, p_{k}$ ) and $p_{1}$ is large enough so that there are (at least) $k$ primes between $p_{1}$ and $f\left(p_{1}, k\right)$. Then $\left\{p_{1}, \ldots, p_{k}\right\}$ forms a prime-field characteristic set.

Proof. We construct the matrix $M(N)$ and the configuration $C(N)$ as in $\S 3$. Theorem 3.3 gives us $\chi(C(N))=\left\{p_{1}, \ldots, p_{k}\right\}$. We will show that $\chi_{p f}(C(N))$ exists (and equals $\left\{p_{1}, \ldots, p_{k}\right\}$ ) by assigning prime-field values to each of $x_{1}, \ldots, x_{n}$ so that no new dependences are created in $M(N)$ modulo any prime $p_{m}(1 \leqslant m \leqslant k)$.

We proceed recursively, assuming that values $c_{1}, c_{2}, \ldots, c_{j-1}$ have been given to $x_{1}, x_{2}, \ldots, x_{j-1}$, respectively, such that no new dependences have been created in the submatrix $\left(M_{1} M\left(c_{1}\right) \cdots M\left(c_{j-1}\right)\right)$ mod $p_{m}$ for any $m$ (i.e., the 0 -subdeterminants of $\left(M_{1} M\left(x_{1}\right) \cdots M\left(x_{j-1}\right)\right)$ exactly match the 0 -subdeterminants of $\left(M_{1} M\left(c_{1}\right) \cdots\right.$ $\left.\left.M\left(c_{j-1}\right)\right)\right)$.

Let $R_{j}$ be the total number of positive integers less than $p_{1}$ which $c_{j}$ cannot be. Then $R_{j}<R_{n}$ if $j<n$, for the selection of $c_{j}$ involves avoiding the roots of fewer polynomials than the selection of $c_{n}$ involves. (There are more subdeterminants in $\left(M_{1} M\left(c_{1}\right) \cdots M\left(c_{n-1}\right) M\left(x_{n}\right)\right)$ than in $\left(M_{1} M\left(c_{1}\right) \cdots M\left(c_{j-1}\right)\right)$.) Thus it will be possible to assign prime-field integer values $c_{j}$ to $x_{j}$ for $1 \leqslant j \leqslant n$ if $R_{n}<p_{1}$. To compute $R_{n}$, we examine the subdeterminants of $M(N)$ which contain at least one column from $M\left(x_{n}\right)$ (where we assume $\left.x_{1}=c_{1}, \ldots, x_{n-1}=c_{n-1}\right)$.

There are $\left(\begin{array}{l}K \\ 3\end{array}\right)-\left({ }^{K-15}\right)$ such subdeterminants, where $K=|M(N)| \approx 14 \cdot \log _{2} N$ $+9 D \cdot k$, and each subdeterminant is (at most) a degree five polynomial in $x_{n}$. Then $c_{n}$ cannot be any of the (at most) 5 roots for each subdeterminant and for each prime $p_{m}$. Hence, $R_{n} \leqslant 5 k\left[\left({ }_{3}^{K}\right)-\left({ }_{3}^{K-15}\right)\right]$. Now

$$
\left(\begin{array}{l}
K \\
3
\end{array}\right)-\left(\begin{array}{c}
K-15 \\
3
\end{array}\right) \approx C_{2} \cdot K^{2} \approx C_{2}\left(\log _{2} N+C_{3} \cdot k\right)^{2} \quad \text { for constants } C_{2} \text { and } C_{3} .
$$

But $\log _{2} N \leqslant k \cdot \log _{2} p_{k}$, so we get $R_{n} \leqslant C_{4} k^{3}\left(\log _{2} p_{k}+C_{3}\right)^{2}$.

Therefore, we can assign a value $c_{n}$ to $x_{n}$ (and hence $c_{j}$ to $x_{j}$ for all $j<n$ ) without introducing new dependences provided $C_{4} k^{3}\left(\log _{2} p_{k}+C_{3}\right)^{2}<p_{1}$, or $p_{k}<f\left(p_{1}, K\right)$ for constants $A=\sqrt{C_{4}} \cdot C_{3}, B=\sqrt{C}_{4}$.

To complete the proof, we need only check that, for $k$ fixed and $p_{1}$ sufficiently large, there are $k$ primes in the interval between $p_{1}$ and $f\left(p_{1}, k\right)$. But this follows easily from the Prime Number Theorem (see e.g., p. 371 of $[5])$. Thus, $\left\{p_{1}, \ldots, p_{k}\right\}$ forms a prime-field set and we are done.

We note that any subset of a prime-field set formed in this fashion will also be a prime-field characteristic set. (Just apply the same construction to the subset.) This proves

COROLlARY 4.2. For any $k>0$, there are infinitely many prime-field characteristic sets containing exactly $k$ primes.

In general, it is unknown (and probably false) whether a subset of a finite prime-field characteristic set is again a prime-field characteristic set. 
5. Cofinite prime-field characteristic sets. It is well known that $\{0\} \cup[p, \infty)_{P}$ forms a cofinite prime-field set for any prime $p\left(\right.$ where $[p, \infty)_{P}$ denotes the set of all primes $\geqslant p$ ). See [2] for details. We wish to determine what other forms cofinite sets can take. In particular, we show that certain finite sets of primes can be excluded from these "upper interval" cofinite sets.

Let $n$ be a positive integer and let $Q$ be an arbitrary set of $k$ primes between $n^{2}$ and $n^{2}+n$ (or $n^{2}+n$ and $(n+1)^{2}$, resp.). Write $p_{i}=n^{2}+r_{i}\left(n^{2}+n+r_{i}\right.$, resp.) for each $p_{i} \in Q$, where $0<r_{i}<n$ for all $i$.

Define the matrix $M(Q)$ to be

$$
\left[\begin{array}{rrrrrrrrrrrrrrrrrrr}
1 & 0 & 0 & 1 & 1 & 1 & 0 & 1 & 0 & 1 & 0 & 1 & 0 & & 1 & 0 & 1 & & 1 \\
0 & 1 & 0 & 1 & 1 & 0 & 1 & -1 & 1 & 0 & 1 & 0 & 1 & \cdots & 0 & 1 & 0 & \cdots & 0 \\
0 & 0 & 1 & 1 & 0 & 1 & 1 & 0 & -1 & 2 & -2 & 3 & -3 & & n & -n & -r_{1} & & -r_{k}
\end{array}\right]
$$

if the primes in $Q$ are between $n^{2}$ and $n^{2}+n$. If each $p_{i} \in Q$ is between $n^{2}+n$ and $(n+1)^{2}$, we define $M^{\prime}(Q)$ be deleting

$$
\left[\begin{array}{l}
1 \\
n \\
0
\end{array}\right]
$$

from $M(Q)$ and adding

$$
\left[\begin{array}{c}
1 \\
0 \\
n+1
\end{array}\right] \text { and }\left[\begin{array}{c}
1 \\
n+1 \\
0
\end{array}\right]
$$

Let $C(Q)\left(C^{\prime}(Q)\right.$, resp.) denote the matroid configuration arising from the column dependences of $M(Q)\left(M^{\prime}(Q)\right.$, resp.) over the integers. We state Theorem 5.1 without proof.

THeorem 5.1. (1) $C(Q)\left(C^{\prime}(Q)\right.$, resp.) is sequentially unique.

(2) $\chi_{p f}(C(Q))=\{0\} \cup\left[n^{2}, \infty\right)_{P}-Q$.

(3) $\chi_{p f}\left(C^{\prime}(Q)\right)=\{0\} \cup\left[n^{2}+n, \infty\right)_{P}-Q$.

Finally, we note that if there are $k$ primes between $n^{2}$ and $n^{2}+n$ (or $n^{2}+n$ and $\left.(n+1)^{2}\right)$, Theorem 5.1 gives us $2^{k}$ prime-field sets, of which only $k+1$ are upper interval sets.

\section{REFERENCES}

1. T. Brylawski, Finite prime-field characteristic sets for planar configurations, Linear Algebra Appl. 46 (1982), 155-176.

2. T. Brylawski and D. Kelly, Matroids and combinatorial geometries, Lecture Notes Ser., University of North Carolina, Chapel Hill, N. C., 1980.

3. T. Brylawski and D. Lucas, Uniquely representable combinatorial geometries, Proceedings of the International Colloquium on Combinatorial Theory, Rome, 1976, pp. 83-104.

4. G. Gordon, Representations of matroids over prime fields, Ph.D. Thesis, University of North Carolina, Chapel Hill, N. C., 1983.

5. G. Hardy and E. Wright, An introduction to the theory of numbers, Oxford Univ. Press, London, 1938, 1945, 1954, 1960, 1979.

6. A. W. Ingleton, Representation of matroids, Combinatorial Mathematics and its Applications (D. J. A. Welsh, ed.), Academic Press, New York, 1971, pp. 149-167. 
7. J. Kahn, Characteristic sets of matroids, J. Loncon Math. Soc. (2) 26 (1982), 207-217.

8. R. Rado, Note on independence functions, Proc. London Math. Soc. (3) 7 (1957), 300-320.

9. R. Reid, Obstructions to representations of combinatorial geometries (unpublished; appears as Appendix in [2]).

10. W. Tutte, Lectures on matroids, J. Res. Nat. Bur. Standards 69B (1965), 1-47.

11. P. Vamos, A necessary and sufficient condition for a matroid to be linear, Matroid Conf. (Brest, 1970).

12. S. S. Wagstaff, Jr., Infinite matroids, Trans. Amer. Math. Soc. 175 (1973), 141-153.

Department of Mathematical Sciences, Williams College, Williamstown, Massachusetts 01267 